\title{
Research on Reliability Measurement Model and Evaluation Method of Distributed Storage Network
}

\author{
Yi'an ZHU ${ }^{1}$, Shuyan LUO $^{1+}$, Peiqi REN ${ }^{2}$ \\ ${ }^{1}$ School of computer science, Northwestern Polytechnical University, Xi'an, China \\ ${ }^{2}$ School of software and microelectronics, Northwestern Polytechnical University, Xi'an, China
}

\begin{abstract}
The structure of distributed storage network is more complex and the number of nodes is increasing. The reliability of the network based on the undirected graph analysis has a certain deviation, because the tasks of each node in the storage network are different, and the upload and download speed is not consistent. Therefore, we introduce the concept of node importance and system entropy and propose a distributed storage network reliability measurement model based on directed graph. Through the calculation comparison, we can see that the reliability evaluation results obtained by this measurement model are closer to the real situation and have great reference value.
\end{abstract}

Keywords: distributed storage, network reliability, measurement model, directed graph, node importance, system entropy.

\section{Introduction}

With the popularity of big data and cloud computing technology, the application of distributed storage network is becoming more and more extensive, and the number of network nodes has increased exponentially. When mass tasks are uploaded and transported in the network, the network topology or the unreasonable distribution of nodes makes the access efficiency of the whole network low and easily leads to the network congestion ${ }^{[1]}$. In order to effectively analyse and evaluate the robustness of distributed storage networks and find out the core nodes in the network, many scholars ${ }^{[2-3]}$ abstract the network topology into undirected graphs. However, the network performance based on undirected graph analysis has a certain deviation due to the different tasks in the real storage network, and the upstream and downlink speed is not consistent. Therefore, on the basis of directed graph, the concept of node importance and system entropy is introduced, and the network structure is abstractly analysed, and a distributed storage network reliability measurement model based on directed graph is proposed.

The model divides the nodes into three categories: Data Storage Node (DMN), Data Transfer Node (DTN) and Data Usage Node (DUN). There are many factors that affect the performance of distributed storage network, such as topology, data transmission path between DMN and DUN. This paper focuses on the modelling and analysis of network topology and node path, try to finds the main reasons that affect the performance of the distributed storage network, and provides a reference for the designers of the system.

\section{Reliability Analysis of Distributed Storage Network}

There is no need to study every node in the analysis of storage performance, but it can abstract a group of DMN or DUN into a single end node cluster and abstract a group of DTN into a single transmission node cluster. End node cluster communicates through several transmission node clusters. As an example of the network shown in Fig. 1, node 1 to 4, node 5 to 6, node 9 to 12 constitute an end node cluster respectively;

\footnotetext{
+ Corresponding author.

E-mail address: 840705@qq.com
} 
node 13 to 16 , node 17 to 20 constitute a transmission node cluster respectively. In a distributed storage network, due to application requirements and physical characteristics of network lines, the transmission speed of nodes will be inconsistent. Therefore, it will lead to deviation of the analysis results if we use the undirected graph to analyse the performance. The directed connected topology is used as the basis for establishing the reliability model of storage network. At first, we introduce the concept of node importance.

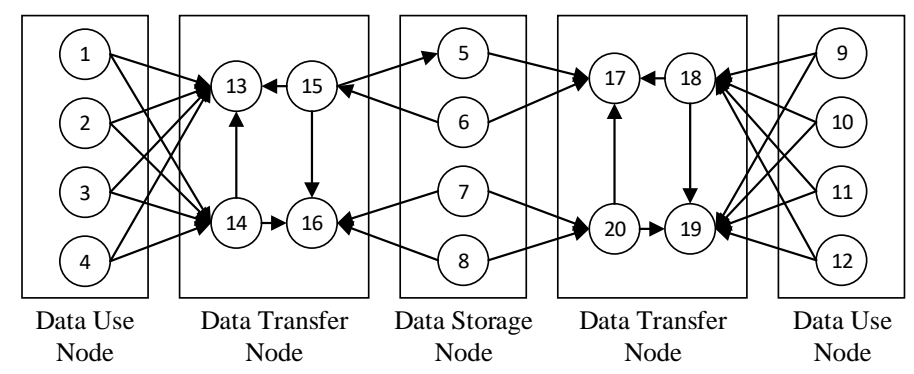

\section{Node Importance}

\subsection{Calculation Method of Existing Node Importance}

Definition 1 Node importance refers to the importance of a node in the whole network.

In storage networks, nodes are not distributed randomly, and some nodes will be at the center of data transmission. Once these nodes fail, the storage capacity of the whole network will be reduced. In addition, even if the nodes of the same type are configured in the storage network, they will affect the storage performance of the system because of their different locations. Therefore, we quantify each node by node importance to find out the impact of network topology on storage performance. In the network topology, it is important to analyze the impact of each node on the performance of the whole network.

At present, many scholars have proposed the calculation methods of the importance of network nodes, such as node degree method, node contraction method, node deletion method, etc. The node degree method uses the node degree as the main basis to measure the importance of the node. This method can only reflect the influence of one node to its adjacent nodes and is lack of integrity. The node contraction method is the integration of a node $v_{i}$ and the $n$ nodes connected to the node, and the network topology after the fusion is calculated. The aggregability of graphs determines the importance of $v_{i}$ in the network. However, since the node has hidden the connection between the node and its adjacent nodes in the process of contraction, it lacks the local characteristics of the network. The node deletion method is to delete the number of trees in the deleted network topology after deleting a node $v_{j}$ and the edge $e_{j}$ connected to it, if the more the spanning tree is reduced, the more nodes are. It's important. This method takes into account the destructiveness of the deletion of the corresponding node to the system network, and the result is more biased towards the impact of the node disappearance on its corresponding local network. Compared with the above methods, the paper [11] proposed a node importance computing method based on node connectivity and local connectivity. This method takes the joint degree and the local connectivity of nodes into consideration as a basis for measuring the importance of nodes. The higher the connectivity is, the smaller the number of nodes and the number of connected edges will be, and the shortest path will be reduced, which proves that the node is more important.

Suppose there is a network $G=(V, E)$ with no direction, no power and no heavy edges. $k_{i}$ is the degree of node $v_{i}$, that is the number of edges associated with the node $v_{i} ; l_{i}$ is the number of node pairs in the network node of the network after the deletion of the node $v_{i}$, and $l_{i}$ is a positive integer between 0 and $k_{i}\left(k_{i}-1\right) / 2$. The $I\left(v_{i}\right)$ shown in Formula 1 is an important degree of node $v_{i}$. Because the leaf node $l_{i}$ may be equal to 0 , the denominator is set $l_{i}+1$ to prevent the denominator being 0 .

$$
I\left(v_{i}\right)=\frac{k_{i}\left(k_{i}-1\right)}{2\left(l_{i}+1\right)}
$$

\subsection{Calculation Method of Node Importance}


The method in Formula 1 assumes that the network topology diagram is an undirected graph, but the undirected graph causes the calculation result to be more theoretical and lack of reference value. We propose a method for computing node importance based on directed connected graph. First, we need to compute the number of node paths in the undirected network, and then compute the node importance.

\section{1) Calculation of the Node Connected Path Number in Undirected Connected Graphs}

The nodes in the undirected graph can be divided into two categories: one is the end node, and another is the communication node.

Definition 2 The End Node (EN) refers to the nodes in the computer network responsible for computing, storage and other functional services. The End node cluster (ENC) refers to the set of end nodes in the same functional cluster. The communication node $(\mathrm{CN})$ refers to the node responsible for data transmission in a computer network. The Communication node cluster (CNC) refers to the set of communication nodes that can be connected to each other.

Taking the Fig. 2 as an example, Node 1, 2, 3, 9, 10, 11 are 6 EN respectively. Node 1, 2, 3 and Node 9, 10, 11 can be divided into ENC respectively. Node 4, 5, 6, 7, 8 are $5 \mathrm{CN}$, and they can be regarded as a CNC.

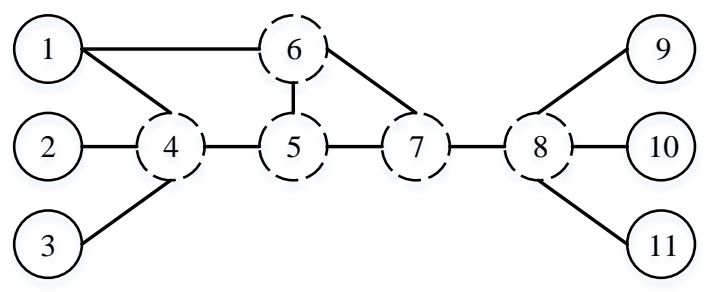

Fig. 2: Undirected connected graph.

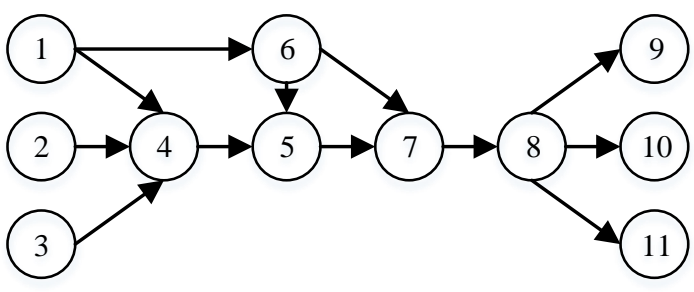

Fig. 3: Directed connected graph.

Definition 3 Suppose there is an unconnected graph G, where $S_{\text {End }}\left\{N_{1}, N_{2}, \ldots N_{n}\right\}, n \geq 1$ is its ENC, $S_{\text {Com }}\left\{C_{1}, C_{2}, \ldots C_{m}\right\}, m \geq 1$ is its CNC, and both $N$ and $C$ can be made up of one or more nodes. For any node $v$, its connected path number (CPN) is the number of paths that can connect to the two different ENC, and it must pass through the node $v$. Among them, the forward path and the reverse path are regarded as the same CPN, and each node has a maximum of one time.

Taking the node 6 in Fig. 2 as an example, the network path includes: 1-6-7-8-9, 1-6-7-8-10, 1-6-7-8-11, 1-6-5-7-8-9, 1-6-5-7-8-10, 1-6-5-7-8-11, 1-4-5-6-7-8-9, 1-4-5-6-7-8-10, 1-4-5-6-7-8-11, 2-4-5-6-7-8-9, 2-45-6-7-8-10, 2-4-5-6-7-8-11, 1-4-5-6-7-8-10, 1-4-5-6-7-8-10. So, the CPN of node 6 is 15.

\section{2) Calculation of the Node Importance in Directed Connected Graph}

We analyze the importance of the existing network nodes and consider the number of node paths. This paper proposes a method for calculating the importance of nodes based on Bidirectional Breadth First Traversal (BBFT).

For the directed connected graph D as shown in Fig. 3. $v_{i}$ is one node in D, and its node importance is $K_{i}$. The calculation method of $K_{i}$ is as follows:

(1) Start with the node $v_{i}$ as the starting node, traverse the forward connection direction of the graph, and we record the number of nodes traversable to $N_{1}$. Node 1 traverses nodes along the forward connection direction of the graph is node $4,6,5,7,8,9,10,11$, and the number of forward traversal nodes is 8 .

(2) Start with the node $v_{i}$ as the starting node and traverse along the reverse direction of the graph, and we record the number of nodes that can traverse $N_{2}$. Node 9 traverses nodes along the reverse connection direction of the graph is node $8,7,5,6,4,1,2,3$, and the number of reverse traversal nodes is 8 .

(3) Calculate the CPN $L_{i}$ of node $v_{i}$, which is the number of paths between nodes and adjacent nodes.

(4) Count the minimum rings number (MRN) $m$ where node $v_{i}$ in. There is a loop consisting of node 1,4 , 5,6 and node 5, 6, 7 formed by the minimum ring in node 5, the MRN of node 5 is 2 .

(5) The importance $K_{i}$ of node $v_{i}$ can be calculated according to Formula 2.

$$
K_{i}=\left(N_{1}+N_{2}\right) * L_{i}-m
$$




\section{Network Reliability Measurement Model}

\subsection{Existing Network Reliability Evaluation Method}

In a distributed storage network, the node importance of most nodes is very small, but the nodes of a few nodes are very important, which indicates that the network is not uniform. The failure of these core nodes may paralyze the entire network. The characteristics reflect the ability of a network architecture to operate normally without disrupting communication when the network faces a sudden catastrophe of the node.

At present, many scholars have put forward the index and the corresponding assessment method to measure the network reliability from different angles. Below are several common measures of survivability.

1) Network Connectivity Method

This paper introduces network connectivity $\mathrm{C}$ to measure network reliability. $\mathrm{C}$ refers to the average path length and the number of connected branches of the connected paths in the network. The smaller the average path length and the fewer the connected branches, the better the connectivity of the network and the higher the reliability of the network.

2) NCF Method

Node connectivity factor (NCF) is put forward by K. T. New Port, which measures the degree of network damage completely. The calculation idea is to remove the connected nodes from a complex network until the remaining nodes are all isolated nodes, and then the average number of statistical removal nodes is used as a measure. The greater the NCF value, the higher the network's ability to resist.

\section{3) Structural Entropy Method}

Based on the anti-damage performance testing of various networks, the paper [12] introduces the concept of structural entropy to measure the damage resistance of complex scale-free networks. For complex scalefree networks, the degree of most nodes is small, and only a few nodes have a larger degree. Therefore, the uniformity of the network is low, and its degree distribution is power law distribution. Therefore, the paper [12] defines the structural entropy of the network as follows:

Define $I_{i}$ as the importance of node $i$, and $K_{i}$ as the degree of $i$, and $N$ as the number of nodes.

$$
I_{i}=\frac{K_{i}}{\sum_{i=1}^{n} K_{i}}
$$

Then the structure entropy of the network is as Formula 4:

$$
E^{\prime}=-\sum_{i=1}^{n} I_{i} * \ln I_{i}
$$

\subsection{Evaluation Method of Network Reliability}

In the storage network, the systems performance may be different because of the different network topology structure when they have the same data processing capability. At the same time, considering that the storage network is a complex directed connected network, and the nodes in the network are not uniform interconnected. The failure of a few core nodes may lead to the paralysis of the entire network. Moreover, the more uneven the network, the greater the overall dependence of the system on the stability of the core node, and the lower the robustness of the network ${ }^{\left[{ }^{*}\right]}$. The paper introduces system entropy as evaluation index in order to measure this characteristic. The more uniform the degree of node distribution, the greater the entropy of the system is.

Definition 4 The system entropy refers to the quantity of the degree of importance distribution of each node in a directed network structure.

The system entropy in the current research of network destruction ability is mainly based on the network topology to describe the undirected graph, and it reflects its ability to resist destruction by calculating the node importance. In this paper, a comprehensive measurement method based on the node importance, the number of nodes and the number of rings in the directed graph is proposed to determine the entropy of the network system, so as to measure the reliability of the network. 
Definition 5 The directed connected path number (DCPN) refers to the path number of connecting a starting node to a termination node in a directed network. In directed connected graphs, the node has export without import is called the starting node, and the node has import without export is called the terminating node.

The starting node uses nodes in the data writing process in the storage network, or the data storage node in the data reading process, that is, the start node of the data stream, the termination node corresponds to the data storage node in the data writing process, or the data use node in the data reading process, that is the end node of the data stream.

Suppose that for directed graph $\mathrm{D}, u_{i}$ is a node in graph $\mathrm{D}$, the calculation steps of system entropy $E$ are as follows:

1) Calculate the node importance $K_{i}$ of the node $u_{i}$.

2) Calculate the number of connected paths $N_{i}$ through the A node. Take Fig. 3 as an example, the connectivity paths of node 5 are 1-4-5-7-8-9, 1-4-5-7-8-10, 1-4-5-7-8-11, 1-6-5-7-8-9, 1-6-5-7-8-10, 1-6-5-78-11, 2-4-5-7-8-9, 2-4-5-7-8-10, 2-4-5-7-8-11, 3-4-5-7-8-9, 3-4-5-7-8-10, 3-4-5-7-8-11, so the number of connectivity paths of node 5 is 12 .

3) Count the minimum rings number (MRN) $m$ where node $u_{i}$ in. Take Fig. 3 as an example, the smallest ring in node 5 consists of rings of 1, 4, 5, 6 and 5, 6, 7 nodes, and MRN of node 5 is 2.

4) The entropy E of the system can be calculated according to Formula 5.

$$
E=\sum_{i=1}^{n} \frac{N_{i}^{*} K_{i}}{m+1}
$$

\section{Performance Analysis}

\subsection{Comparison of Importance}

For the directed connected graph as shown in Fig. 3, the calculation of the importance degree $K_{i}$ of the corresponding node by this method is compared with the node importance calculated by the node importance $K_{i}^{\prime}$ calculation method mentioned in the paper [12], and the results are shown in Table 1.

Table 1: Node importance table

\begin{tabular}{|c|c|c|c|c|c|c|}
\hline Node & $\mathrm{N}_{1}$ & $\mathrm{~N}_{2}$ & $\mathrm{~L}_{\mathrm{i}}$ & $\mathrm{m}$ & $K_{i}$ & $K_{i}^{\prime}$ \\
\hline 1 & 8 & 0 & 12 & 1 & 95 & 0.5 \\
\hline 2 & 7 & 0 & 6 & 0 & 42 & 0 \\
\hline 3 & 7 & 0 & 6 & 0 & 42 & 0 \\
\hline 4 & 6 & 3 & 18 & 1 & 161 & 3 \\
\hline 5 & 5 & 5 & 21 & 2 & 208 & 0.75 \\
\hline 6 & 6 & 1 & 15 & 2 & 103 & 0.75 \\
\hline 7 & 4 & 6 & 24 & 1 & 239 & 1.5 \\
\hline 8 & 3 & 7 & 24 & 0 & 240 & 6 \\
\hline 9 & 0 & 8 & 8 & 0 & 64 & 0 \\
\hline 10 & 0 & 8 & 8 & 0 & 64 & 0 \\
\hline 11 & 0 & 8 & 8 & 0 & 64 & 0 \\
\hline
\end{tabular}

We can see that the order of node importance $K_{i}$ by our method is $8,7,5,4,6,1,9,10,11,2,3$, and the $K_{i}$ ' calculated by node importance function method are $8,4,7,5,6,1,9,10,11,2,3$. The importance of node 5 and node 6 is the same, and the importance of the node 4 is higher than them. As shown in Fig. 3, deleting node 7 and 8 will make the $\operatorname{ENC}(1,2,3)$ unconnected to the $\operatorname{ENC}(8,9,11)$, and deleting node 5 will make the $\operatorname{ENC}(2,3)$ unconnected to the $\operatorname{ENC}(8,9,11)$, and it also reduces the connectivity between node 1 and $\operatorname{ENC}(8,9,11)$; deleting node 4 will make the $\operatorname{ENC}(2,3)$ unconnected to the $\operatorname{ENC}(8,9,11)$; deleting node 6 will not make the $\operatorname{ENC}(1,2,3)$ unconnected to the $\operatorname{ENC}(8,9,11)$, but it will reduce the connectivity between the two node clusters. Therefore, node 4, 5, 6, 7, 8 importance rank should be $8,7,5,4,6$. The ranking results calculated by our method agree with the actual situation. 


\subsection{The Comparison of Entropy}

Taking Fig. 3 as an example, the comparison of calculation results between the structural entropy $E$ ' by Formula 4 and system entropy $E$ by Formula 5 are shown in Table 2. We can see that the structural entropy of the Fig. 3 is 28.1969 , and the system entropy of the Fig. 3 is 8794.5.

Table 2: The comparison between structural entropy and system entropy

\begin{tabular}{|c|c|c|c|c|}
\hline \multirow{2}{*}{$\begin{array}{c}\text { Node } \\
\text { Num }\end{array}$} & Structural Entropy Method & \multicolumn{3}{|c|}{ System Entropy Method } \\
\cline { 2 - 5 } & $K_{i}^{\prime}$ & $K_{i}$ & CPN & MR \\
\hline 1 & 2 & 95 & 9 & 1 \\
\hline 2 & 1 & 42 & 3 & 0 \\
\hline 3 & 1 & 42 & 3 & 1 \\
\hline 4 & 4 & 161 & 9 & 2 \\
\hline 5 & 3 & 208 & 12 & 2 \\
\hline 6 & 3 & 103 & 6 & 1 \\
\hline 7 & 3 & 239 & 15 & 0 \\
\hline 8 & 4 & 240 & 15 & 0 \\
\hline 9 & 1 & 64 & 5 & 0 \\
\hline 10 & 1 & 64 & 5 & \\
\hline 11 & $\mathbf{2 8 . 1 9 6 9}$ & 64 & 5 & $\mathbf{8 7 9 4 . 5}$ \\
\hline Entropy & & & & \\
\hline
\end{tabular}

In order to better explain the importance of each node and the impact on the whole network, the following two schemes are given to change the network topology, and the importance of each node is judged by observing the structure entropy of the system.

1) Remove the node 6 of Fig. 3 in the directed connected graph as shown in Fig. 4. Obviously, the stability of node 1,2,3 to node 9,10,11 is reduced. Once the node 4, 5, 7 or 8 fail, Fig. 4 will not guarantee the connectivity of the whole graph.

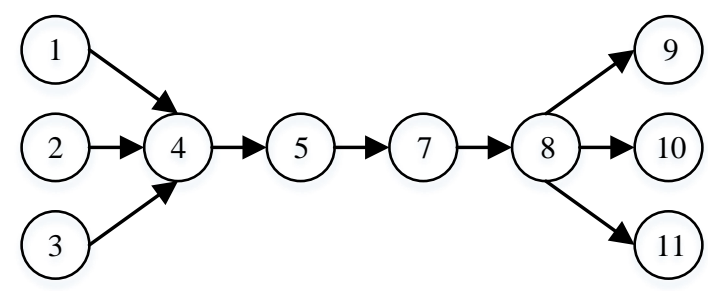

Fig. 4: Directed connected graph.
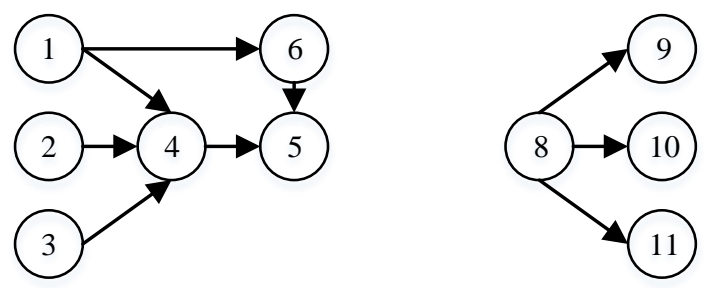

Fig. 5: Directed connected graph.

The structural entropy $E_{2}^{\prime}$ is 24.7448 , and the system entropy $E_{2}$ is 3294 . It can be seen that our method is better (shown in Table 3 Schemes 1), and it can effectively reflect the reduction of the data flow path from nodes $1,2,3$ to node $9,10,11$ after the node 6 is removed. The decrease of entropy value leads to the decrease of network reliability.

2) Remove the node 7 of Fig. 3 in the directed connected graph as shown in Fig. 5. Obviously, the stability of node $1,2,3$ to node $9,10,11$ is reduced. The removal of node7 will not guarantee the connectivity of the whole graph. The node 1, 2, 3 will not be able to communicate with node 9, 10, 11 normally.

The structural entropy $E_{3}^{\prime}$ is 24.3393 , and the system entropy $E_{3}$ is 70 . It shown in Table 3 Schemes 2 . It can be seen that the $E_{3}^{\prime}$ is lower than $E^{\prime}$ and $E_{2}^{\prime}$. However, the system entropy is directly reduced to 70 by our method, and the decrease is great. Compared with Fig. 3 and Fig. 5, we can see that with the removal of node 6 , there is no effective path between node $1,2,3$ to node $8,9,11$. It is obvious that structural entropy method pays attention to the connectivity problem of undirected graphs, although it can reflect the reliability of the system, but the method cannot effectively reflect the consequences of the core node failure to the 
system in the directed connected graph. But the system entropy method takes into account the selfcharacteristics of the directed connected graph, and it can better reflect the reliability of network when the failure of the important node.

Table 3: The comparison between structural entropy and system entropy in two schemes

\begin{tabular}{|c|c|c|c|c|c|c|c|c|}
\hline \multirow{3}{*}{$\begin{array}{l}\text { Node } \\
\text { Num }\end{array}$} & \multicolumn{4}{|c|}{ Scheme 1} & \multicolumn{4}{|c|}{ Scheme 2} \\
\hline & \multirow{2}{*}{$\begin{array}{c}\begin{array}{c}\text { Structural } \\
\text { Entropy } \\
\text { Method }\end{array} \\
K_{i}^{\prime}\end{array}$} & \multicolumn{3}{|c|}{ System Entropy Method } & \multirow{2}{*}{$\begin{array}{c}\text { Structural } \\
\text { Entropy } \\
\text { Method }\end{array}$} & \multicolumn{3}{|c|}{ System Entropy Method } \\
\hline & & $K_{i}$ & $\mathrm{CPN}$ & MRN & & $K_{i}$ & $\mathrm{CPN}$ & MRN \\
\hline 1 & 1 & 21 & 3 & 0 & 2 & 5 & 2 & 1 \\
\hline 2 & 1 & 21 & 3 & 0 & 1 & 2 & 1 & 0 \\
\hline 3 & 1 & 21 & 3 & 0 & 1 & 2 & 1 & 0 \\
\hline 4 & 4 & 81 & 9 & 0 & 4 & 11 & 3 & 1 \\
\hline 5 & 2 & 81 & 9 & 0 & 2 & 19 & 4 & 1 \\
\hline 6 & - & - & - & - & 1 & 1 & 1 & 1 \\
\hline 7 & 2 & 81 & 9 & 0 & - & - & - & - \\
\hline 8 & 4 & 81 & 9 & 0 & 1 & 3 & 1 & 0 \\
\hline 9 & 1 & 21 & 3 & 0 & 1 & 1 & 1 & 0 \\
\hline 10 & 1 & 21 & 3 & 0 & 2 & 1 & 1 & 0 \\
\hline 11 & 1 & 21 & 3 & 0 & 2 & 1 & 1 & 0 \\
\hline Entropy & 24.7448 & & 3294 & & 24.3393 & & 70 & \\
\hline
\end{tabular}

\subsection{Network Optimization Analysis}

Considering the actual engineering applications, the bottleneck of the network can be compared through the node importance. There are two ways to solve the bottleneck: increasing the node or increasing the link. The advantage of the former is that the nodes with excessive load pressure in the network can reduce the importance of one or more nodes by diverting the network, thus increasing the network survivability, but the disadvantage is that the additional overhead is increased and the cost of the network erection is increased; the latter does not need to increase the additional resources, but the net is not added. The load of the collaterals will not decrease. In view of the above two situations, the network survivability is analyzed by the model. Fig. 6 shows the scheme of adding nodes, and Fig. 7 shows the scheme of adding link.

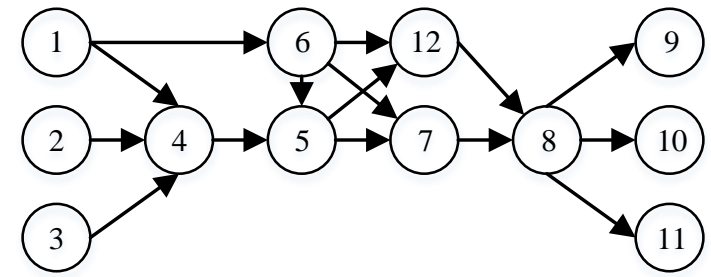

Fig. 6: Scheme 1: increase the node 12.

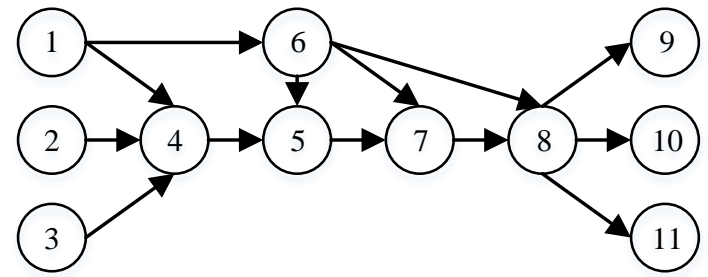

Fig. 7: Scheme 2: increase the link between 6 and 8.

According to the directed connected graph in Fig. 6 and Fig. 7, the entropy of the two schemes can be calculated as shown in Table 4. We can see that increasing the number of nodes increases the entropy of the system more than that of the link scheme and enhances the reliability of the network.

\section{Concluding}

At present, the reliability of distributed storage network is evaluated by node importance and system entropy, but this method has some limitations. First, how to cluster effectively and scientifically is the problem to consider when the network scale is very complex. Secondly, although the model takes into account the different functions of the node, it does not take the upload/download speed and the 
reading/writing speed as a reference factor, that is, the physical configuration of all the nodes is the same, but this is an obvious ideal state. Third, in real environment, the probability of the arrival of each node is subordinate to a certain mathematical distribution, not a linear distribution. Therefore, when calculating the node importance, it is necessary to consider the effect of the task frequency on the network reliability.

\section{Acknowledgements}

The project supported by the Aviation Science Fund (Grant 20150753010), the Civil Aircraft Project (Grant XJZ-2015-D-76), and the major and key project of the Shaanxi Province key research and development plan (Grant 2016MSZD-G-8-1).

Table 4: Structural entropy $E_{3}$

\begin{tabular}{|c|c|c|c|c|c|c|}
\hline \multirow{2}{*}{$\begin{array}{l}\text { Node } \\
\text { Num }\end{array}$} & \multicolumn{3}{|c|}{ Scheme 1} & \multicolumn{3}{|c|}{ Scheme 2} \\
\hline & $\begin{array}{c}\text { Node } \\
\text { Importance } K_{i}\end{array}$ & $\begin{array}{c}\text { Connected } \\
\text { Path Number }\end{array}$ & $\begin{array}{l}\text { The Minimum } \\
\text { of Rings }\end{array}$ & $\begin{array}{c}\text { Node } \\
\text { Importance } K_{i}\end{array}$ & $\begin{array}{l}\text { Connected } \\
\text { Path Number }\end{array}$ & $\begin{array}{l}\text { The Minimum } \\
\text { of Rings }\end{array}$ \\
\hline 1 & 323 & 18 & 1 & 167 & 12 & 1 \\
\hline 2 & 144 & 6 & 0 & 84 & 3 & 0 \\
\hline 3 & 144 & 6 & 0 & 84 & 3 & 0 \\
\hline 4 & 539 & 18 & 1 & 323 & 9 & 1 \\
\hline 5 & 722 & 24 & 4 & 388 & 12 & 2 \\
\hline 6 & 428 & 12 & 4 & 249 & 9 & 3 \\
\hline 7 & 477 & 15 & 3 & 328 & 15 & 2 \\
\hline 8 & 790 & 30 & 2 & 449 & 18 & 1 \\
\hline 9 & 216 & 10 & 0 & 120 & 6 & 0 \\
\hline 10 & 216 & 10 & 0 & 120 & 6 & 0 \\
\hline 11 & 216 & 10 & 0 & 120 & 6 & 0 \\
\hline 12 & 477 & 15 & 3 & - & - & - \\
\hline Entropy & \multicolumn{3}{|c|}{31936.3} & \multicolumn{3}{|c|}{12912.75} \\
\hline
\end{tabular}

\section{Reference}

[1] Gopal Sekar, Ayyappan Govindan, Muthu Ganapathi Subramanian. Stability Analysis of Single Server Retrial Queuing System with Erlang Service[C]. Proceedings of the 6th International Conference on Queueing Theory and Network, 2011. 155-161.

[2] R. Kalyanaraman. A single server batch service finite source queue with feedback. Proceedings of the 6th International Conference on Queueing Theory and Network Applications, 2011. 84-88.

[3] A. M. Dos Santos, M. L. De Almeida, G. A. Mendes, et al. Generalized scale-free hemophilic network[J]. International Journal of Modern Physics C, 2015, 26(09):362-.369.

[4] Mahessar A W, Waqas A, Mehmood N, et al. Analyzing the Weighted Dark Networks using Scale-Free Network Approach[J]. Wseas Transactions on Computers, 2015, 14(73):748-759.

[5] XiangMin Guan, XueJun Zhang, Yanbo Zhu, et al. An efficient routing strategy on spatial scale-free networks[J]. International Journal of Modern Physics C, 2014, 25(7):809-820.

[6] Section I. A Multilevel Simplification Algorithm for Computing the Average Shortest-Path Length of Scale-Free Complex Network[J]. Journal of Applied Mathematics, 2014, 2014(4):1-6.

[7] Li Q. Towards Efficient Routing to Addresses and Names in Computer Communication Networks[J]. Electronic Theses \& Dissertations, 2014(4):1-6.

[8] Netapp I. Network Storage Management at Scale Using Service Level Objectives[J]. 2015.

[9] Tang Q, Sun S, Yang C, et al. Learning Scale Free Network by Node Specific Degree Prior[J]. Computer Science, 2015:2247-2255.

[10] Zhang Y, Ma J. Power-law index and its application based on the degree of distribution and scale-free network 
evolution[J]. Wseas Transactions on Mathematics, 2014, 13:505-515.

[11] Y.Zhang, J.L.Wang. Reliability Evaluation of the Network Storage System Based on Block Diagrams. Computer Science, 2010, 37(6): 102-105.

[12] L.G.Zhu, Z.S.Yang, H.Y.Luo. Comprehensive evaluation technology for networked storage system. Computer Engineering and Applications, 2010, 46(36):61-66.

[13] Liu Z, Jiang C, Wang J, et al. The node importance in actual complex networks based on a multi-attribute ranking method[J]. Knowledge-Based Systems, 2015, 84:56-66.

[14] Fan W, Ping H, Liu Z. Multi-attribute node importance evaluation method based on Gini-coefficient in complex power grids[J]. IET Generation Transmission \& Distribution, 2016, 10(9):2027-203. 